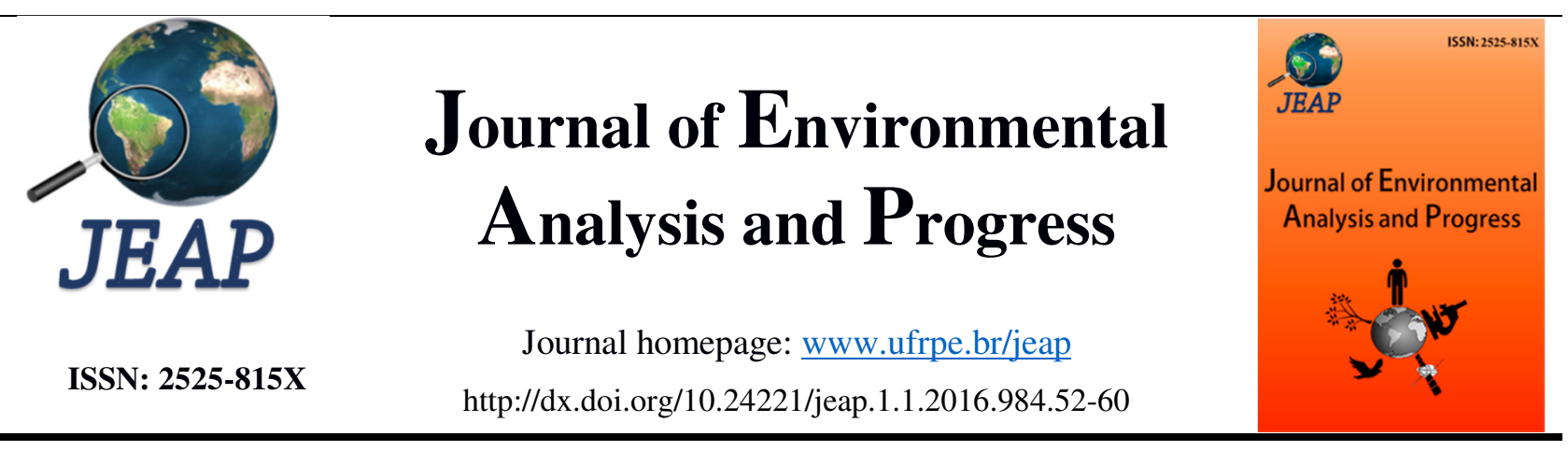

\title{
Comportamento hidrodinâmico de solos em cultivos de vazante no semiárido de Pernambuco (Brasil)
}

\section{Hydrodynamic behavior of soils in recession agriculture in semiarid of Pernambuco State (Brazil)}

Eduardo Soares de Souza ${ }^{\mathrm{a}}$, Antonio Celso Dantas Antonino ${ }^{\mathrm{b}}$, André Maciel Netto ${ }^{\mathrm{b}}$, Rodolfo Marcondes Silva Souza ${ }^{\mathrm{b}}$, Manuella Virginia Salgueiro Gondim ${ }^{\mathrm{b}}$, Valmir Felix de Lima ${ }^{\mathrm{b}}$, José Romualdo de Sousa Lima ${ }^{c}$, Edevaldo Miguel Alves ${ }^{\mathrm{d}}$, Artur Paiva Coutinho ${ }^{\mathrm{d}}$, Willames de Albuquerque Soares ${ }^{\mathrm{e}}$

a Unidade Acadêmica de Serra Talhada, Universidade Federal Rural de Pernambuco-UFRPE. Avenida Gregório Ferraz Nogueira S/N, José Tomé de Souza Ramos, CEP: 56909-535, Serra Talhada-PE, Brasil. E-mail: eduardo.ssouza@ufrpe.br.

${ }^{\mathrm{b}}$ Departamento de Energia Nuclear, Universidade Federal de Pernambuco. Avenida Professor Luiz Freire, 1000, Cidade Universitária, CEP: 50740-545, Recife-PE, Brasil. E-mail: acda@ufpe.br, amnetto@ufpe.br, rodolfo.marcondes@ufpe.br, manuellavirginia@yahoo.com.br, atrazina@gmail.com.

${ }^{\mathrm{c}}$ Unidade Acadêmica de Garanhuns, UFRPE. Avenida Bom Pastor S/N, Boa Vista, CEP: 55292-270, Garanhuns-PE. Email: romualdo.lima@ufrpe.br.

${ }^{d}$ Centro Acadêmico do Agreste, Universidade Federal de Pernambuco. Rodovia BR-104, Km 59, s/n, Nova Caruaru, CEP: 55002-970, Caruaru-PE. E-mail: edevaldofisica@gmail.com, arthur.coutinho@ yahoo.com.br.

e Universidade de Pernambuco (UPE), Escola Politécnica de Pernambuco, Rua Benfica, 455, Madalena, Recife-PE (Brasil). 50720-001. E-mail: was@ poli.br.

\section{A R T I CLE I N F O}

Recebido 28 Sep2016

Aceito 30 Sep2016

Publicado 13 Oct 2016

\begin{abstract}
A B S T R A C T
Hydrodynamic behavior of soils in recession agriculture was evaluated through of the soil water characteristic curve $\theta(\mathrm{h})$, hydraulic conductivity as a function of the soil water potential $K(h)$, sorptivity $S$, and characteristic pore radius $\lambda_{m}$, in two Fluvents soils at the recession emerging zone of Cajueiro and Flocos reservoirs, in Tuparetama, PE. The soil of Cajueiro shows a little transition of texture and dry bulk density, while the soil from Flocos has an evident transition from texture and bulk density with depth. The curves $\theta(\mathrm{h})$ were obtained by fitting the model proposed by van Genuchten to field data (obtained with tensiometers and a neutron scattering probe), and to laboratory data (obtained with a Richards pressure chamber). Experiments on the disk infiltrometer ( $0.08 \mathrm{~m}$ diameter base) were performed for obtaining $\mathrm{K}$ and $\mathrm{S}$ values, at the depths of $0,0.20,0.40,0.60$ and $0.80 \mathrm{~m}$, applying four water potentials. The $\theta(\mathrm{h})$ curves showed good agreement for potentials ranging from -30 to $-10 \mathrm{kPa}$, indicating the good quality of the experimental data. The soil hydrodynamic heterogeneity in Flocos may be explained by texture and dry bulk density and in Cajueiro this heterogeneity is induced by the pore size distribution. The diameter of the infiltrometer that was used together with the applied pressures, have allowed characterizing the porous matrix of the clay layer of Flocos. Keywords: Hydraulic conductivity, sorptivity, disc infiltrometer, texture.
\end{abstract}

\section{R E S U M O}

O comportamento hidrodinâmico de solos em cultivo de vazante foi analisado por meio das curvas de retenção $\theta(\mathrm{h})$, da condutividade hidráulica $K(\mathrm{~h})$, da sorvidade $S$ e do raio característico de poros $\lambda_{\mathrm{m}}$, determinados em dois NeossolosFlúvicos das 
vazantes dos açudes Cajueiro e Flocos, em Tuparetama, PE. O solo de Cajueiro apresenta uma pequena transição de textura e de densidade do solo, já o de Flocos apresenta uma evidente transição dessas propriedades em profundidade. As curvas de $\theta(h)$ foram obtidas pelo ajuste da equação de van Genuchten aos valores de campo (por meio de medidas de tensiômetros e de sonda de nêutrons) e de laboratório (por meio da "câmara de pressão de Richards"). Para determinação de K e S, foram realizados ensaios com o infiltrômetro a disco $(0,08$ m diâmetro da base), com quatro tensões de fornecimentos, nas profundidades de 0, 0,20, 0,40, 0,60 e 0,80 m. As curvas de $\theta(\mathrm{h})$ mostraram boa concordância na faixa de potencial de $-30 \mathrm{a}-10 \mathrm{kPa}$, indicando coerência dos dados experimentais. A heterogeneidade hidrodinâmica do solo de Flocos é explicada pela textura e densidade do solo e em Cajueiro é induzida pela distribuição dos tamanhos dos poros. O diâmetro do infiltrômetro, combinado às tensões aplicadas, permitiu caracterizar a matriz porosa da camada argilosa do solo de Flocos.

Palavras-Chave:Condutividade hidráulica, sorvidade, infiltrômetro a disco, textura.

\section{Introdução}

A questão dos recursos hídricos é sem dúvida uma das mais importantes para o nordeste do Brasil, em particular, estando relacionada à utilização da água na agricultura de sequeiro, de vazante ou de irrigação. No semiárido nordestino, as áreas dos vales, baixadas ou baixios, e as áreas planas ou suavemente onduladas que lhes são adjacentes, são as que têm maior potencial de produção agrícola, em função de uma maior disponibilidade hídrica. Para elas convergem às águas das bacias, seja na forma dos riachos intermitentes ou do escoamento subterrâneo (Antonino et al., 2004).

Parte destas áreas é ocupada por milhares de açudes construídos no semiárido para armazenar água durante a estação de chuvas. Finda esta estação, os açudes vão secando, pela evaporação, e possivelmente pela redistribuição ou drenagem interna no perfil do solo e/ou pelo uso da água para os mais diversos fins. À medida que os açudes secam, vão emergindo áreas nas suas bordas que são chamadas de vazantes e que são largamente usadas para a agricultura. A agricultura de vazante consiste em cultivar nestas bordas ou margens, em declive suave, à medida que a água vai baixando. A cultura utiliza apenas a água presente no solo para realizar o seu ciclo e produz em plena época de seca (Antonino et al., 2005).

Existem três maneiras de utilizar a água dos açudes para a produção agrícola: cultura de vazante, irrigação a montante e a irrigação a jusante das barragens. A vazante se destaca, dentre as alternativas de utilização hídrica dos açudes para fins agrícolas, como um método de baixo custo, que não requer implantação de sistemas de adução e irrigação (Antonino \& Audry, 2001).

O procedimento de vazante pode ser classificado como método de subirrigação, no qual a umidade do perfil é parcialmente ou totalmente dependente da presença de um lençol freático raso e das propriedades relacionadas à ascensão capilar (Montenegro et al., 2003).

A infiltrometria a disco com uma sucção controlada (Zhang, 1997; Tamoh \& Maslouhi, 2004; Ventrella et al., 2005; Ndiaye et al., 2005; Souza et al., 2006; Yoon et al., 2007) é um método que permite realizar medidas no campo para determinar os parâmetros hidráulicos do solo, em condições próxima à saturação, tais como a condutividade hidráulica e a sorvidade.

Em relação à irrigação, a disponibilidade de conhecimentos científicos e tecnológicos permite a sua avaliação econômica. No entanto, a falta de conhecimento sobre o cultivo de vazante, ou seja, do comportamento hidrodinâmico dos solos em cultivo de vazante, não possibilita uma avaliação das suas potencialidades (Antonino \& Audry, 2001).

Este estudotem por objetivo analisar o comportamento hidrodinâmico de dois solos das vazantes dos açudes de Cajueiro e de Flocos, em Tuparetama, Pernambuco, por meio da curva de retenção, condutividade hidráulica, sorvidade, textura, estrutura e pelas dimensões de poros hidraulicamente funcionais.

\section{Material e Métodos}

Os experimentos para a determinação das propriedades hidráulicas foram realizados em duas parcelas de dois diferentes solos das vazantes dos açudes de Cajueiro e Flocos, localizados no município de Tuparetama, PE (7036'0' S e $\left.37^{\circ} 18^{\prime} 45^{\prime}, \mathrm{W}\right)$.

Na parcela do açude de Cajueiro encontrase um solo caracterizado como Neossolo Flúvico (Santos et. al., 2013). Este perfil apresenta-se bastante arenoso, com transição de textura e de densidade do solo, entre as camadas, relativamente pequenas (Tabela 1). Na análise granulométrica, as frações de argila e de silte foram determinadas por sedimentação, após dispersão com hexametafosfato de sódio utilizando-se o método 
da pipeta, e a fração areia foi separada por peneiramento (Loveland \& Whalley, 2000). A densidade do solo foi determinada utilizando-se o método do anel volumétrico (Campbell \& Henshall, 2000).
O solo de Flocos, também caracterizado como Neossolo Flúvico (Santos et al., 2013), evidencia aumento de densidade do solo com a profundidade, apresentando camada superficial argilosa seguida de camadas arenosas (Tabela 1).

Tabela 1. Análise granulométrica e densidade dos solos de Cajueiro e de Flocos.

\begin{tabular}{|c|c|c|c|c|}
\hline Camada & $\rho$ & Areia & Silte & Argila \\
\hline $\mathbf{m}$ & kg.m ${ }^{-3}$ & & g. $\mathrm{kg}^{-1}$ & \\
\hline \multicolumn{5}{|c|}{ Solo de Cajueiro } \\
\hline $0,05-0,15$ & $1.460 \pm 40$ & 838 & 69 & 93 \\
\hline $0,15-0,25$ & $1.470 \pm 20$ & 858 & 54 & 88 \\
\hline $0,35-0,45$ & $1.460 \pm 30$ & 926 & 25 & 50 \\
\hline $0,50-0,70$ & $1.410 \pm 30$ & 873 & 46 & 81 \\
\hline $0,70-0,80$ & $1.390 \pm 00$ & 903 & 35 & 62 \\
\hline \multicolumn{5}{|c|}{ Solo de Flocos } \\
\hline $0,05-0,15$ & $1.430 \pm 30$ & 303 & 256 & 441 \\
\hline $0,15-0,25$ & $1.420 \pm 10$ & 380 & 207 & 413 \\
\hline $0,35-0,45$ & $1.580 \pm 50$ & 852 & 57 & 91 \\
\hline $0,50-0,70$ & $1.580 \pm 20$ & 902 & 25 & 73 \\
\hline $0,70-0,80$ & $1.630 \pm 30$ & 859 & 46 & 95 \\
\hline
\end{tabular}

As curvas de retenção dos solos foram obtidas pelo ajuste da equação de van Genuchten (1980) aos valores obtidos em campo, por meio de medidas tensiométricas e neutrônicas (utilizandose uma sonda de nêutrons NARDEUX - 20) (Antonino \& Audry, 2001), e aos valores obtidos em laboratório, determinados em amostras de solo indeformadas utilizando-se a "câmara de pressão de Richards".

Nas profundidades de 0, 0,20, 0,40, 0,60 e $0,80 \mathrm{~m}$, foram realizados ensaios de infiltração com água, com infiltrômetro a disco conforme descrito em Antonino et al. (2001), aplicando-se tensões de $0,-0,3,-0,6 \mathrm{e}-1,2 \mathrm{kPa}$ com três repetições por pressão.

\section{Determinação de parâmetros hidráulicos segundo Zhang (1997)}

Zhang (1997) propôs uma equação com dois termos para descrever o processo de infiltração a partir do infiltrômetro a disco, para qualquer tempo de infiltração:

$$
I=C_{1} t^{1 / 2}+C_{2} t
$$

sendo $\quad \mathrm{C}_{1} \quad \mathrm{e} \quad \mathrm{C}_{2}$ parâmetros respectivamente relacionados com a sorvidade $\mathrm{S}\left(\mathrm{h}_{0}\right)$ e a condutividade hidráulica $\mathrm{K}\left(\mathrm{h}_{0}\right)$ do solo, pelas expressões:

$$
\begin{aligned}
& C_{1}\left(h_{0}\right)=A_{1} S\left(h_{0}\right) \\
& C_{2}\left(h_{0}\right)=A_{2} S\left(h_{0}\right)
\end{aligned}
$$

sendo $A_{1}$ e $A_{2}$ coeficientes adimensionais; $h_{0}(\leq 0)$ é o valor da pressão usada no infiltrômetro a disco durante o processo de infiltração.

A sorvidade, $\mathrm{S}\left(\mathrm{h}_{0}\right)$, e a condutividade hidráulica, $\quad \mathrm{K}\left(\mathrm{h}_{0}\right)$, foram determinadas respectivamente pelas Eqs. (2) e (3). A partir de experimentos de infiltração, $\mathrm{C}_{1}$ e $\mathrm{C}_{2}$ são obtidos pelo ajuste da Equação (1) aos dados da infiltração acumulada. Conhecendo-se a curva característica da umidade do solo, $\mathrm{A}_{1}$ e $\mathrm{A}_{2}$ podem ser calculados pelo método van Genuchten-VG proposto por Zhang (1997), descrito a seguir.

\section{Método van Genuchten-VG}

A equação proposta por van Genuchten (1980) é dada por:

$$
\Theta(h)=\frac{\theta-\theta_{r}}{\theta_{S}-\theta_{r}}=\left[1+|\alpha h|^{n}\right]^{-m}(4)
$$

com, $\mathrm{m}=1-1 / \mathrm{n}(\mathrm{n}>1) \mathrm{e}$ 


$$
K(h)=K_{s} \Theta^{\chi}\left[1-\left(1-\Theta^{1 / m}\right)^{m}\right]^{2}(5)
$$

sendo, $\theta \mathrm{r}$ e $\theta_{\mathrm{S}}$ correspondentes à umidade volumétrica residual e de saturação respectivamente, $\mathrm{n}$ e $\alpha$ são parâmetros de ajuste, Ks é a condutividade hidráulica à saturação e $\chi$ é um parâmetro de conectividade de poros, com o valor de 0,5 conforme sugerido por Mualem (1976).

Os coeficientes $\mathrm{A}_{1}$ e $\mathrm{A}_{2}$, respectivos as equações (2 e 3), são expressos conforme Zhang (1997):

$$
\begin{gathered}
A_{1}=\frac{1,4 b^{0,5}\left(\theta_{0}-\theta_{i}\right)^{0,25} \exp \left[3(n-1,9) \alpha h_{0}\right]}{\left(\alpha r_{0}\right)^{0,15}} \\
A_{2}=\frac{11,65\left(n^{0,1}-1\right) \exp \left[2,92(n-1,9) \alpha h_{0}\right]}{\left(\alpha r_{0}\right)^{0,91}} \\
n \geq 1,9 \\
A_{2}=\frac{11,65\left(n^{0,1}-1\right) \exp \left[7,5(n-1,9) \alpha h_{0}\right]}{\left(\alpha r_{0}\right)^{0,91}} \\
n<1,9
\end{gathered}
$$

sendo n e $\alpha$ parâmetros da curva característica de umidade do solo, $\mathrm{h}_{0}(\leq 0)$ a tensão aplicada pelo infiltrômetro, $r_{0}$ o raio do infiltrômetro, $\theta_{0}$ o conteúdo de água em $\mathrm{h}_{0}, \theta_{\mathrm{i}}$ o conteúdo de água inicial e $b$ um fator de forma que pode variar de 0,5 a $\pi / 4$, para o qual foi adotado o valor de 0,55 (White \& Sully, 1987).

\section{Escalas características}

A escala de comprimento capilar, $\lambda_{c}$ (White \& Sully, 1987) e o raio característico de poros, $\lambda_{m}$ (Philip, 1987) foram determinados respectivamente pelas equações:

$$
\lambda_{c}=\frac{b S_{0}^{2}}{\left(\theta_{0}-\theta_{i}\right) \Delta K}
$$

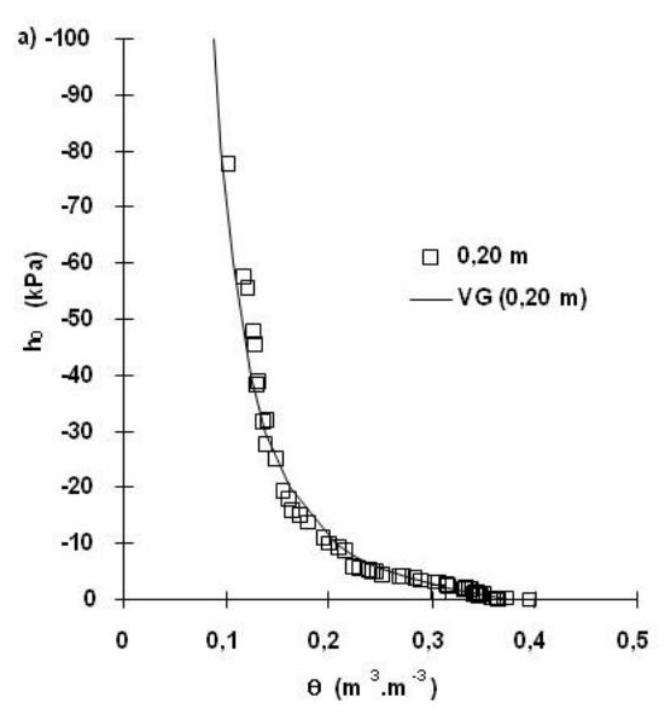

sendo $\Delta \mathrm{K}=\mathrm{K}_{0}-\mathrm{K}_{\mathrm{i}}$; considerando $\mathrm{K}_{0} \gg>\mathrm{K}_{\mathrm{i}}$, tem-se $\Delta \mathrm{K}=\mathrm{K}_{0}$.

$$
\lambda_{m}=\frac{\sigma}{\rho_{a} g \lambda_{c}}
$$

sendo $\sigma$ a tensão superficial $\left(0,0719 \mathrm{~kg} \cdot \mathrm{s}^{-2}\right), \rho_{\mathrm{a}}$ a densidade da água $\left(1000 \mathrm{~kg} \cdot \mathrm{m}^{-3}\right)$ e "g" a aceleração da gravidade $\left(9,81 \mathrm{~m} \cdot \mathrm{s}^{-2}\right)$.

A escala de comprimento capilar (Equação 9) representa a importância relativa das forças capilares em relação à gravidade, quando a água é transmitida de uma fonte mantida a $\theta_{0}$ através do solo, com umidade inicial $\theta_{\mathrm{i}}$. O raio característico de poros (Equação 10) define a dimensão média dos poros que participam do processo de infiltração. Quanto maior for o raio característico, $\lambda_{\mathrm{m}}$, maior é o efeito da gravidade em comparação ao da capilaridade.

A concentração de raio característico de poros $\lambda_{\mathrm{m}}, \mathrm{C}_{\lambda \mathrm{m}}$ (número de poros $\mathrm{m}^{-2}$ ), é estimada usando-se a lei de Poiseuille para fluxo em um tubo capilar, a partir da seguinte expressão:

$$
C_{\lambda m}=\frac{8 \mu K}{\rho_{a} g \pi \lambda_{m}^{4}}
$$

sendo $\mu$ a viscosidade dinâmica da água $(0,00089$ kg. $\mathrm{m}^{-1} \cdot \mathrm{s}^{-1}$, em $\mathrm{T}=25^{\circ} \mathrm{C}$ ).

\section{Resultados}

Açude Cajueiro

As curvas de retenção do solo de Cajueiro, em campo, foram obtidas para potenciais entre -80 e $0 \mathrm{kPa}$ (para a profundidade de $0,20 \mathrm{~m}$ ) (Figura 1a) e entre -10 e $0 \mathrm{kPa}$ (para as profundidades de 0,40 , 0,60 e $0,80 \mathrm{~m}$ ) (Figura 1b). Para a faixa de potenciais observada de $-10 \mathrm{a}-3 \mathrm{kPa}$ nas curvas de $\mathrm{h}(\theta)$, observa-se que a profundidade de $0,40 \mathrm{~m}$ foi a que apresentou a maior variação de umidade.

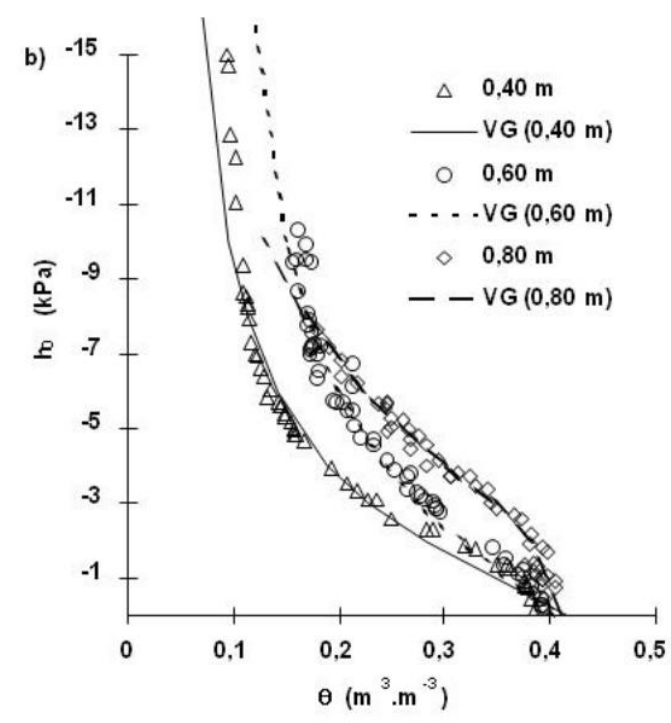


Figura 1. Curvas características da umidade do solo na área de vazante do açude Cajueiro obtidas no campo e em laboratório, ajustadas com a equação de van Genuchten $(1980)(\mathrm{VG})$, para as profundidades de a) 0,20 m e b) $0,40,0,60$ e $0,80 \mathrm{~m}$.

A condutividade hidráulica $(K)$ e a sorvidade (S) aumentam com a profundidade (Figura 2a, b). Entretanto, os valores de $\mathrm{K}$ e de $\mathrm{S}$ diminuem, quando a sucção aumenta. $\mathrm{Na}$ superfície, o valor de raio característico de poros $\left(\lambda_{m}\right)$ hidraulicamente funcionais (Equação 10) foi de $0,12 \mathrm{~mm}$ na saturação, indicando uma presença de macroporos $\left(\lambda_{\mathrm{m}}>0,1 \mathrm{~mm}\right)$. A $0,20 \mathrm{~m}$ de profundidade o valor de $\lambda_{\mathrm{m}}$ aumenta para $0,4 \mathrm{~mm}$, assim como K e S (Figura 2c). Para profundidades maiores que $0,20 \mathrm{~m}$, os valores de $\lambda_{\mathrm{m}}$ diminuem na saturação e K e S aumentam. Isto é explicado pelo fato de que com a redução do tamanho dos poros ocorre um consequente aumento na quantidade sorvidade e da condutividade hidráulica (Figuras $2 a$ e $2 b)$.

Tabela 2. Parâmetros das equações de van Genuchten (1981) para o solo de Cajueiro.

\begin{tabular}{cccc}
\hline Camada & $\boldsymbol{\theta}_{\mathbf{S}}$ & $\boldsymbol{\alpha}$ & $\mathbf{n}$ \\
\hline $\mathbf{m}$ & $\mathbf{m}^{3} \mathbf{~ m}^{-3}$ & $\left(\mathbf{x ~ \mathbf { 1 0 } ^ { - 2 } )} \mathbf{c m}^{-\mathbf{1}}\right.$ & \\
\hline $0,15-0,25$ & 0,376 & 4,21 & 1,269 \\
$0,35-0,45$ & 0,416 & 5,90 & 1,407 \\
$0,50-0,70$ & 0,404 & 4,70 & 1,342 \\
$0,70-0,80$ & 0,411 & 2,14 & 1,330 \\
\hline
\end{tabular}
2c e 2d), contribuindo assim para o aumento da

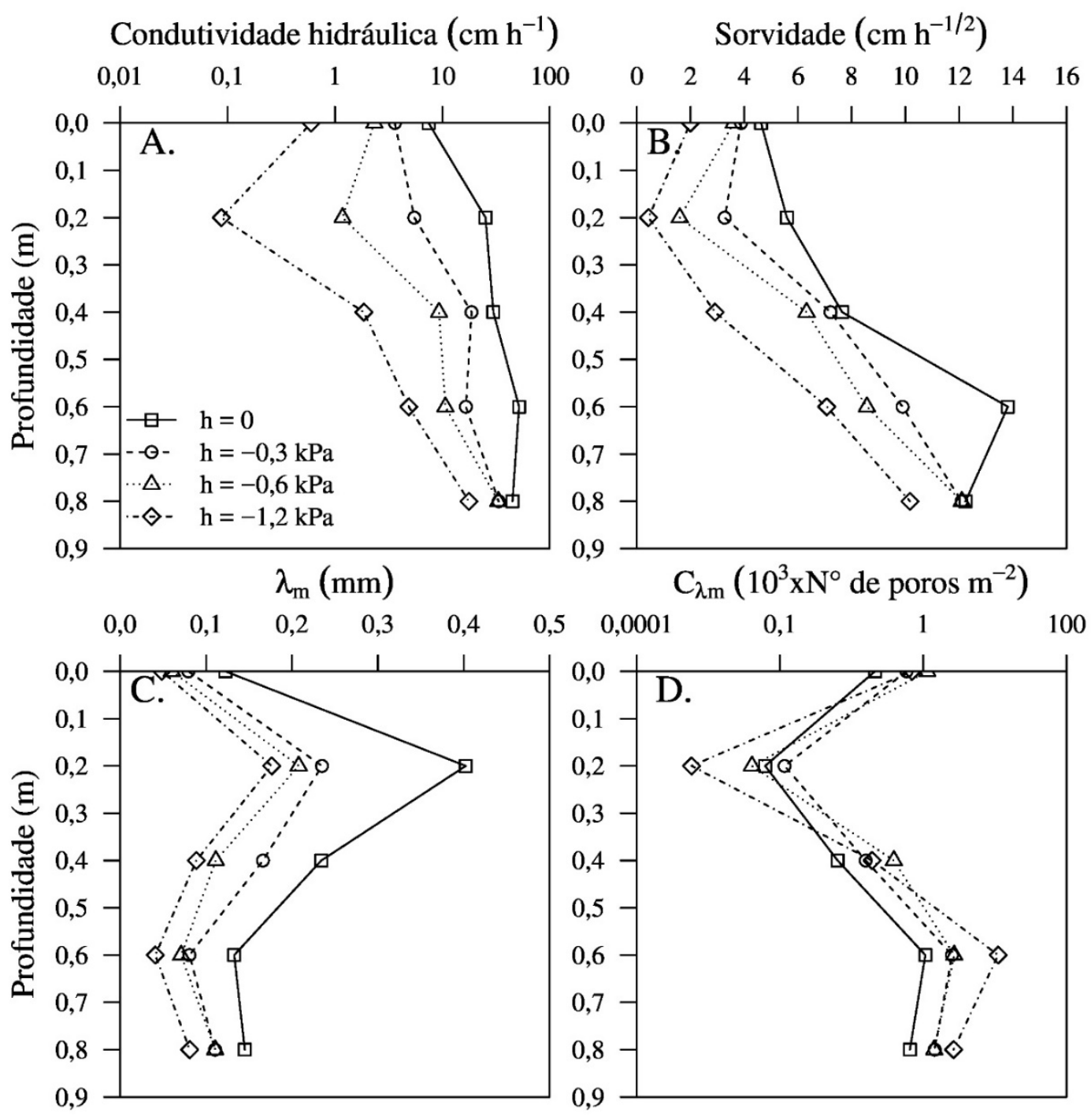

Figura 2. Perfis da condutividade hidráulica (a), da sorvidade (b) e do raio característico de poros, $\lambda_{\mathrm{m}}$ (c) e a concentração de raio característico de poros $C_{\lambda m}(d)$ para as tensões de fornecimento da água de $0,-0,3,-0,6$ e $-1,2 \mathrm{kPa}$ do solo na área de vazante do açude Cajueiro. 
$\mathrm{O}$ escoamento em meio poroso saturado compreende o fluxo tanto nos microporos como nos macroporos (Othmer et al., 1991). Quando o solo se torna não saturado, isto é, com o aumento da sucção, a macroporosidade contribui menos no escoamento da água no solo. Isto corresponde a uma redução mais acentuada da condutividade hidráulica, sobretudo, na profundidade de $0,20 \mathrm{~m}$, onde $\lambda_{m}$ é maior e $C_{\lambda m}$ é menor. Uma redução insignificante na condutividade hidráulica ocorre quando os valores de $\lambda_{\mathrm{m}}$ são menores, como se observa nas profundidades de 0,40 a $0,80 \mathrm{~m}$. Este comportamento hidráulico heterogêneo é reflexo da heterogeneidade da estrutura dos poros do perfil do solo.

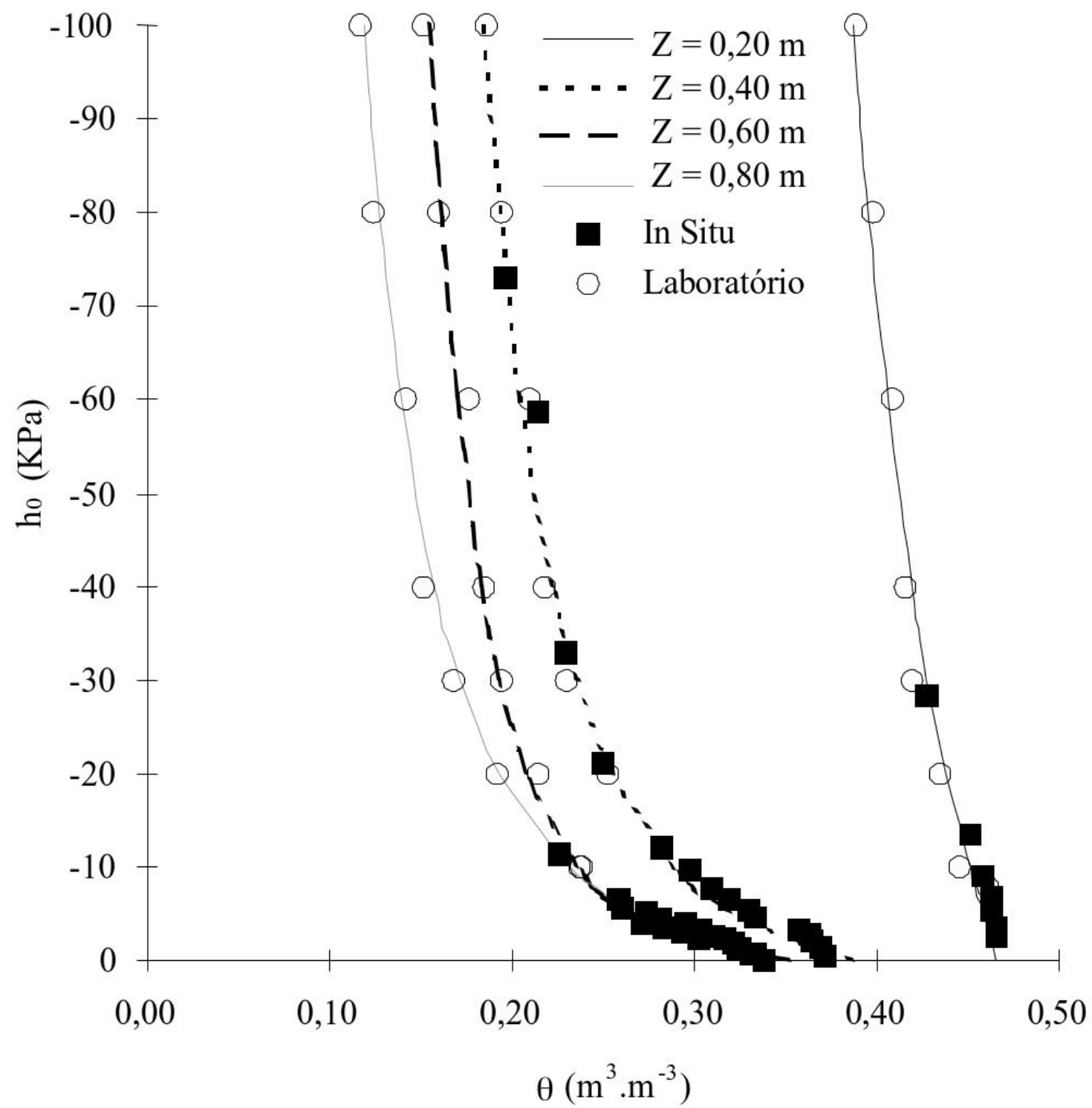

Figura 3. Curvas características da umidade do solo na área de vazante do açude de Flocos, obtidas no campo e em laboratório, ajustadas com a equação de van Genuchten (1980), para as profundidades de 0,20, 0,40, 0,60 e $0,80 \mathrm{~m}$.

Os coeficientes de determinação $\mathrm{R}^{2}$ também indicaram bons ajustes para as diferentes profundidades com valores superiores a 0,960 . Os

\section{Açude Flocos}

A curva de retenção do solo foi obtida pelos dois métodos, no campo e em laboratório, na faixa de potenciais entre -100 e $0 \mathrm{kPa}$ (Figura 3 ). A boa concordância entre as medidas dos métodos na faixa de potencial comum, de -30 a $-10 \mathrm{kPa}$, mostra bem a coerência dos dados experimentais. Observa-se uma menor variação da umidade volumétrica para a camada argilosa, na profundidade de $0,20 \mathrm{~m}$, em relação às camadas mais arenosas, nas profundidades de 0,40, 0,60 e $0,80 \mathrm{~m}$, na faixa de potenciais explorados. 
Journal of Environmental Analysis and Progress V. 01 N. 01 (2016) 52-60

A condutividade hidráulica $(K)$ e a sorvidade (S) na saturação apresentam um comportamento uniforme para a camada de $0-0,40$ $\mathrm{m}$, seguida de um aumento brusco na profundidade de $0,60 \mathrm{~m}$ e após uma redução na profundidade de $0,80 \mathrm{~m}$ (Figura 4ab). Os valores de $\mathrm{K}$ e de $\mathrm{S}$ diminuem quando a sucção aumenta.

Os valores do raio de poros hidraulicamente funcionais $\left(\lambda_{\mathrm{m}}\right)$ indicam a ausência de macroporos entre a superfície do solo e a profundidade de $0,20 \mathrm{~m}$, a presença demacroporos na profundidade de $0,40 \mathrm{~m}$, seguida de um forte aumento de macroporos de 0,40 a 0,60 $\mathrm{m}$ de profundidade (Figura 4c). Os valores de macroporos e a forte variação entre 0,40 e $0,80 \mathrm{~m}$ de profundidade confirmam que a camada mais permeável do solo encontra-se a $0,60 \mathrm{~m}$ de profundidade.

Tabela 3. Parâmetros das equações de van Genuchten (1981) para o solo de Flocos.

\begin{tabular}{cccc}
\hline Profundidade & $\boldsymbol{\theta}_{\mathbf{s}}$ & $\boldsymbol{\alpha}$ & $\mathbf{n}$ \\
\hline $\mathbf{m}$ & $\mathbf{m}^{\mathbf{3}} \mathbf{m}^{-3}$ & $\left(\mathbf{x} \mathbf{1 0}^{-2}\right) \mathbf{c m}^{-\mathbf{1}}$ & \\
\hline $0,15-0,25$ & 0,466 & 0,13 & 1,391 \\
$0,35-0,45$ & 0,383 & 1,29 & 1,817 \\
$0,50-0,70$ & 0,364 & 4,28 & 1,618 \\
$0,70-0,80$ & 0,343 & 2,35 & 2,419 \\
\hline
\end{tabular}

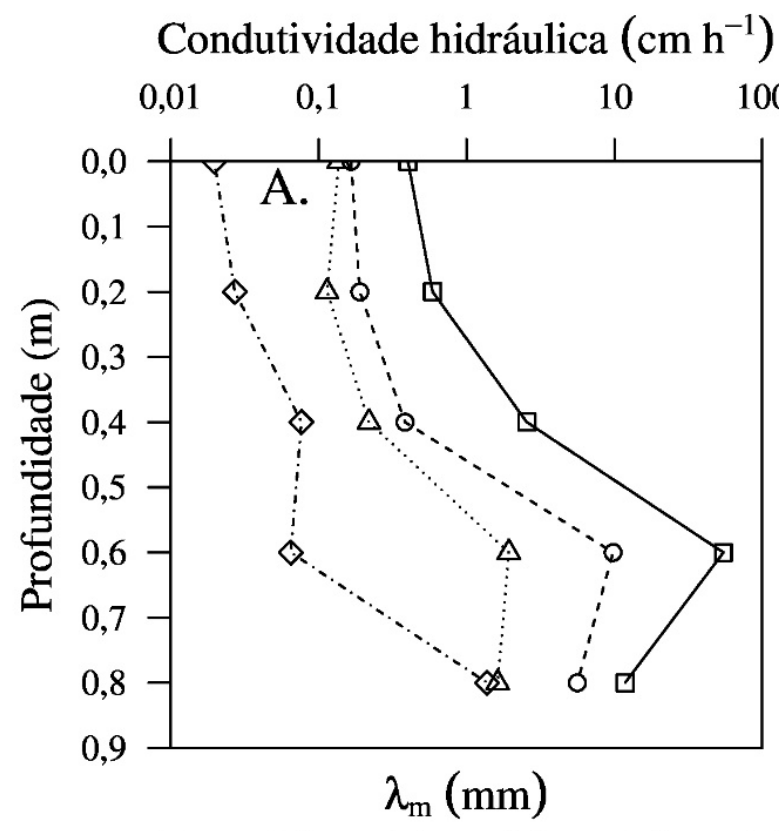

\section{Sorvidade $\left(\mathrm{cm} \mathrm{h}^{-1 / 2}\right)$}
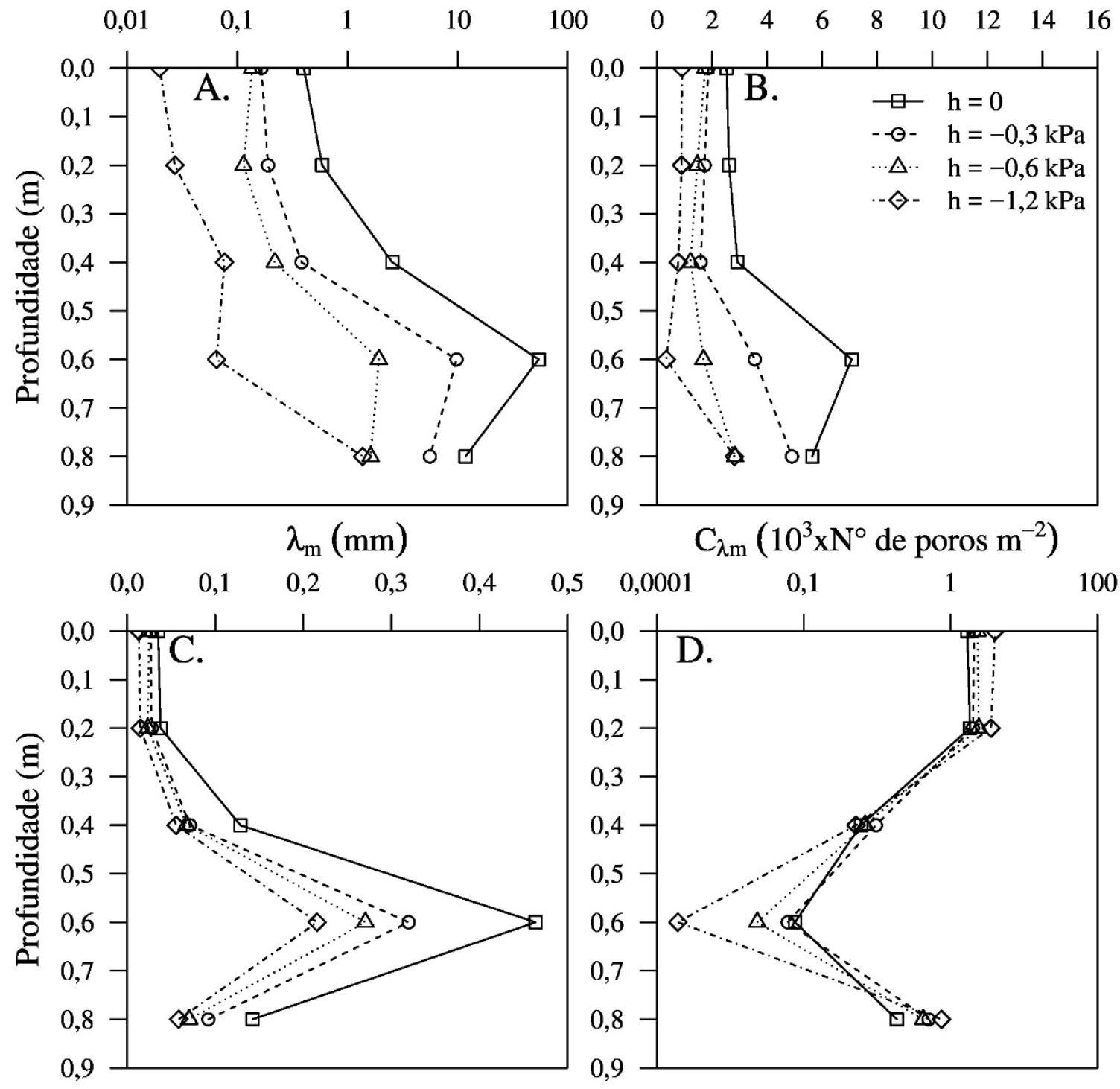

Figura 4. Perfis da condutividade hidráulica (a), da sorvidade (b) e do raio característico de poros, $\lambda_{\mathrm{m}}$ (c) e a concentração de raio característico de poros, $\mathrm{C}_{\lambda \mathrm{m}}(\mathrm{d})$ para as tensões de fornecimento da água de $0,-0,3,-0,6$ $-1,2 \mathrm{kPa}$ do solo de Flocos. 
No perfil do solo de Flocos identificam-se duas camadas com textura e densidade contrastantes (Tabela 1). A camada de 0 a $0,25 \mathrm{~m}$ apresenta-se argilosa, com uma densidade do solo da ordem de $1430 \mathrm{~kg} \mathrm{~m}^{-3}$, aumentando a um valor médio de $1600 \mathrm{~kg} \mathrm{~m}^{-3}$ para a camada subjacente de 0,35 a $0,80 \mathrm{~m}$, na qual a textura é arenosa $(85 \%$ de areia em média).

$\mathrm{O}$ teor de materiais finos (silte e argila) na camada de 0 a 0,20 m explica os baixos valores de condutividade hidráulica (K), de sorvidade (S) e de raio característico de poros $\left(\lambda_{\mathrm{m}}\right)$ medidos em todas as sucções.

A $0,6 \mathrm{~m}$ de profundidade, $\mathrm{o}$ raio característico de poros hidraulicamente funcionais diminuiu com o aumento da sucção. Os valores médios de $\lambda_{\mathrm{m}}$ foram de $0,22 \mathrm{~mm}$ para $\mathrm{h}=-1,2 \mathrm{kPa}$ e de $0,46 \mathrm{~mm}$ para $\mathrm{h}=0$. Esses valores estão de acordo com os reportados em outros trabalhos (Souza et al., 2006; Souza et al., 2007; Souza et al., 2008; Mubarak et al., 2009; Schwen et al., 2011). Apesar de apresentar valores médios de $\lambda_{\mathrm{m}}$ menores, a profundidade de $0,8 \mathrm{~m}$ é considerada uma boa condutora devido à quantidade de poroshidraulicamente ativos por unidade de área $\left(\mathrm{C}_{\lambda \mathrm{m}}\right)$, que variou de $0,19 \times 10^{3}$ até $0,75 \times 10^{3} \mathrm{~N}^{\circ}$ poros $\mathrm{m}^{-2}$; assim, embora o valor de $\lambda_{\mathrm{m}}$ tenha aumentado na profundidade de $0,6 \mathrm{~m}$, a quantidade de poros hidraulicamente ativos foi aproximadamente 24 vezes menor (para $\mathrm{h}=0$ ) e quase 4.000 vezes menor (para $h=-1,2 \mathrm{kPa}$ ) que na profundidade de $0,8 \mathrm{~m}$.

\section{Conclusões}

1. A heterogeneidade hidrodinâmica do perfil do solo da área de vazante do açude Cajueiro não é explicável pelos perfis de textura e de densidade do solo mas, sim pela distribuição dos tamanhos dos poros hidraulicamente ativos.

2. $\mathrm{Na}$ área de vazante do açude Flocos, a heterogeneidade hidrodinâmica do perfil é explicada pelos perfis de textura e de densidade do solo.

\section{Agradecimentos}

Ao CNPq (Processos: 485086/2013-1, 306635/2014-3, 307641/2013-9; 312218/2013-3), pela concessão de bolsa e dos recursos necessários ao desenvolvimento deste.

\section{Referências}

ANGULO-JARAMILLO, R.; VANDERVAERE, J. P.; ROULIER, S.; THONY, J. L.; GAUDET, J. P.; VAUCLIN, M. 2000. Field Measurement of soil surface hydraulic properties by disc and ring infiltrometers: a review and recent developments. Soil Till. Res., v.55, p.1-29.
ANTONINO, A. C. D.; ANGULO-JARAMILLO, R.; SOUZA, E. S.; NETTO, A. M.; CARNEIRO, C. J. G.; MONTENEGRO, A. A. A. 2001. Determinação da condutividade hidráulica e da sorvidade de um solo com infiltrômetro a disco. Rev. Bras. Eng. Agric. Amb., v.5, p.247-253.

ANTONINO, A. C. D.; AUDRY, P. 2001. Utilização de água no cultivo de vazante no semiárido do nordeste do Brasil. Tópicos Especiais em Recursos Hídricos e Tecnologia Ambiental, n.02, Recife: Editora da UFPE.

ANTONINO, A. C. D.; ANGULO-JARAMILLO, R.; HAMMECKER, C.; NETTO, A. M.; MONTENEGRO, S. M. G. L.; LIRA, C. A. B. O.; CABRAL, J. J. S. P. 2004. A simplified water transfer model of the reservoir-ebb tide system, including preferential flow, in the semi-arid region in Northeastern Brazil.J. Hydrol., v.287, n.1-4, p.147-160.

ANTONINO, A. C. D.; HAMMECKER, C.; MONTENEGRO, S. M. G. L.; NETTO, A. M.; ANGULO-JARAMILLO, R.; LIRA, C. A. B. O. 2005. Subirrigation of land bordering small reservoirs in the semi-arid region in the Northeast of Brazil: Monitoring and Water balance. Agric. Water Manag., v.73, n.2, p.131-147.

CAMPBELL, D. J.; HENSHALL, J. K. 1991.Bulk density. In: SMITH K. A.; MULLINS C. E. Soil analysis - physical methods. pp.315-348. New York: Marcel Dekker.

SANTOS, H. G.; JACOMINE, P. K. T.; ANJOS, L. H. C.; OLIVEIRA, V. A.; LUMBRERAS, J. F.; COELHO, M. R.; ALMEIDA, J. A.; CUNHA, T. J. F.; OLIVEIRA, J. B. 2013. Sistema brasileiro de classificação de solos. 3.ed. rev. e ampl. Brasília: Embrapa. 353p.

LOVELAND, P. J.; WHALLEY, R. W. 2000.Particle size analysis. In: SMITH K. A.; MULLINS C. E. Soil analysis - physical methods. 2nd ed., pp.281-314. New York: Marcel Dekker.

MONTENEGRO, A. A. A.; MONTENEGRO, S. M. G. L.; ANTONINO, A. C. D.; MONTEIRO, A. L. N. 2003. Evolução da zona saturada em agricultura de vazante: Açude Cajueiro, Pernambuco. Rev. Bras. Rec. Hidr., v.38, n.2, p.9399.

MUALEM, Y. A. 1976. A new model for predicting the hydraulic conductivity of 
unsaturated porous media.Water Resour. Res., v.12, p.513-522.

MUBARAK, I.; MAILHOL, J. C.; ANGULOJARAMILLO, R.; RUELLE, P.; BOIVIN, P.; KHALEDIAN, M. 2009. Temporal variability in soil hydraulic properties under drip irrigation.Geoderma, v.150, p.158-165.

NDIAYE, B.; ESTEVES, M.; VANDERVAERE, J. P.; LAPETITE, J. M.; VAUCLIN, M. 2005. Effect of rainfall and tillage direction on the evolution of surface crusts, soil hydraulic properties and runoff generation for a sandy loam soil. J.Hydrol., v.307, p.294-311.

OTHMER, H.; DIEKKRUGER, B.; KUTILEK, M. 1991.Bimodal porosity and unsaturated hydraulic conductivity.Soil Sci., v.152, p.139-150.

PHILIP, J. R. 1987. The quasi-linear analysis, the scattering analog, and other aspects of infiltration and seepage. In: FOK, Y. S. Infiltration development and application. pp.1-27. Honolulu: Water Resources Research Center.

SCHWEN, A.; BODNER, G.; SCHOLL, P.; BUCHAN, G. D.; LOISKANDL, W. 2011.Temporal dynamics of soil hydraulic properties and the water-conducting porosity under different tillage. Soil Till. Res., v.113, p.89-98.

SOUZA, E. S.; ANTONINO, A. C. D.; ANGULOJARAMILLO, R.; NETTO, A. M. 2008. Caracterização hidrodinâmica de solos: Aplicação do método Beerkan. Rev. Bras. Eng. Agric. Amb., v.12, p.128-135.

SOUZA, E. S.; ANTONINO, A. C. D.; LIMA, J. R. S.; GOUVEIA NETO, G. C.; SILVA, J. M.; SILVA, I. F. 2007. Efeito do encrostamento superficial nas propriedades hidráulicas de um solo cultivado. Rev. Bras. Ciênc. Agrar., v.2, p.69-74.
SOUZA, E. S.; ANTONINO, A. C. D.; NETTO, A. M.; CABRAL, J. J. S. P.; ANGULOJARAMILLO, R.; ROULIER, S. 2006. Análise do regime transitório da infiltração tridimensional sob sucção controlada. Rev. Bras. Rec. Hidr., v.11, p.513.

TAMOH, K.; MASLOUHI, A. 2004. Mesures in situ par infiltrométrie des propriétés hydrodynamiques des sols de Mnasra (Maroc). Comptes Rendus Geoscience, v.336, p.535-545.

TOWNEND, J.; REEVE, M. J.; CARTER A. D. 2000. Water release characteristic. In: SMITH K. A.; MULLINS C. E. Soil analysis - physical methods. 2nd ed. pp.95-140. New York: Marcel Dekker.

VAN GENUCHTEN, M. TH. 1980. A closed-form equation for predicting the hydraulic conductivity of unsaturated soils. Soil Sci. Soc. Am. J., v.44, p.892-898.

VENTRELLA, D.; LOSAVIO, N.; VONELLA, A. V.; LEIJ, F. J. 2005. Estimating hydraulic conductivity of a fine-textured soil using tension infiltrometry. Geoderma, v.124, p.267-277.

WHITE, I.; SULLY, M. J. 1987. Macroscopic and microscopic capillary length and times scales from field infiltration. Water Resour. Res., v.23, p.15141522.

YOON, Y.; KIM, J. G.; HYUN, S. 2007.Estimating soil water retention in a selected range of soil pores using tension disc infiltrometer data. Soil Till. Res., v.97, p.107-116, 2007.

ZHANG, R. 1997. Determination of soil sorptivity and hydraulic conductivity from the disc infiltrometer.Soil Sci. Soc. Am. J., v.61, p.10241030. 